\title{
Regulation of Apoptosis and Radiation Sensitization in Lung Cancer Cells via the Sirt1/NF-kB/Smac Pathway
}

\author{
Kaihua Ji Xiaohui Sun Yang Liu Liqing Du Yan Wang Ningning He \\ Jinhan Wang Chang Xu Qiang Liu
}

Tianjin Key Laboratory of Radiation Medicine and Molecular Nuclear Medicine, Department of Radiobiology, Institute of Radiation Medicine of Chinese Academy of Medical Science \& Pecking Union Medical College, Tianjin, PR China

\section{Key Words}

Sirt1 • NF-kB • Smac • Apoptosis • Radiation sensitization

\begin{abstract}
:
Background/Aims: SirT1, a conserved $\mathrm{NAD}^{+}$-dependent deacetylase, has been implicated in modulating cell survival and stress responses, and it appears to play an important role in tumorigenesis and cancer resistance to chemoradiotherapy. The mechanism of SirT1 in cancer chemoradiotherapy remains to be further elucidated, which could provide potential targets for cancer therapy. Methods: We performed colony formation, immunofluorescence microscopy, flow cytometry, RNA interference, and western blotting assays to determine whether SirT1 regulates radiation sensitization and which mechanisms and/or pathways it takes in lung cancer cell lines A549 and H460. Results: Initially, the expression of SirT1 was found to be negatively correlated with radiosensitivity in lung cancer cell lines A549 and H460. RNA interference with siSirT1 against SirT1 specifically reduced SirT1 expression and induced radiosensitivity both in A549 and H460 cell lines. In contrast, the radiosensitivity was significantly reduced once SirT1 was activated by resveratrol. Immunofluorescence assay and apoptosis analysis indicated that the effect of SirT1 on the radiosensitivity observed in the A549 and H460 cell lines was mainly achieved by regulating DNA damage repair and apoptosis processes. Furthermore, the expression of SirT1 negatively modulated the expression of apoptosis-related protein NF- $\mathrm{KB}$ and its downstream regulator of Smac. Conclusion: Our results indicate that SirT1 regulates apoptosis and radiation sensitization in lung cancer cell lines A549 and H460 via the SirT1/ NF-kB/Smac pathway.

(C) 2018 The Author(s)

Published by S. Karger AG, Basel
\end{abstract}

\section{Introduction}

Lung cancer, which is one of the most common cancers, results in about 1.3 million deaths each year worldwide and has been identified as the first and second leading cause of cancer deaths in men and women, respectively [1-4]. Clinical treatment of lung cancer

Kaihua Ji and Xiaohui Sun contributed equally to this work.

\begin{tabular}{ll}
\hline Qiang Liu & Tianjin Key Laboratory of Radiation Medicine and Molecular Nuclear Medicine, Dept. of \\
and Chang Xu & Radiobiology, Inst. of Radiation Medicine of Chinese Academy of Medical Science, Tian- \\
& jin 300192, (China); E-Mail liuqiang@irm-cams.ac.cn, xuchang@irm-cams.ac.cn
\end{tabular}




\section{Cellular Physiology Cell Physiol Biochem 2018;48:304-316 \\ \begin{tabular}{c|c} 
DOI: 10.1159/000491730 & O 2018 The Author(s). Published by S. Karger AG, Basel \\
wwww.karger.com/cpb
\end{tabular}}

Ji et al.: Sirt1/ NF-אM/Smac Pathway for Radiation Sensitization

includes surgical resection combined with chemotherapy or radiotherapy. However, lung cancer patients have lower 5-year survival rates with limited efficacy of cancer treatment compared with other cancers [5]. A major contributor to this limited efficacy is the intrinsic or acquired resistance of lung cancer to radiation and/or chemotherapy [6]. Thus, there is a need for molecular approaches focused on the sensitization of lung cancer cells and the elucidation of the basal molecular mechanisms in lung cancer.

Sirtuin, the mammalian silent information regulator 1 family, is a molecular family that includes seven members (SirT1 to SirT7, respectively) and shares extensive homologies with the silent information regulator 2 (sir2) gene in yeast [7]. SirT1, located in the cell nucleus, is the closest homologue of sir2 and a well-documented member of the sirtuin family [8]. Many studies have shown that SirT1 plays a major role in the regulation of cell survival and stress responses, such as responses to oxidative stress, nutrient starvation or calorie restriction, and DNA damage [7, 9-13]. SirT1, as a NAD ${ }^{+}$-dependent histone deacetylase, has a remarkably wide spectrum of substrates that not only interacts with and regulates histone $\mathrm{H} 1, \mathrm{H} 3$ and $\mathrm{H} 4$, but also with non-histone substrates such as nuclear factor- $\kappa \mathrm{B}$ family, tumor suppressor p53, Ku70, fork-head transcription factors (i.e., FOXOS), and DNA repair factor E2F1 proteins $[7,10,14-16]$. Therefore, SirT1 plays many biological roles, including the inhibition of cell differentiation, cell cycle regulation, apoptosis inhibition and tumorigenesis $[17,18]$. Notably, the expression of SirT1 is upregulated in several cancers including leukemia, colon cancer, prostate cancer, and lung cancer [7, 8, 19, 20]. However, the exact effect of SirT1 in cancer development is still unclear. Several reports have shown that the overexpression of SirT1 is correlated with cancer resistance to chemotherapy, ionizing radiation (IR), and DNA damage agents [8, 21-24]. Sun et al. showed that downregulation of SirT1 could induce apoptosis and enhance radiation sensitization in A549 lung cancer cells [8]. All these research findings suggest that SirT1 could be a potential target in cancer therapy. Unfortunately, the exact molecular mechanism responsible for the observed function remains largely unexplored.

Second mitochondria-derived activator of caspases (Smac), which is also a direct inhibitor of apoptosis protein binding protein with low PI (DIABLO), is a mitochondrial intermembrane space protein that regulates apoptosis [25]. In apoptotic cells, Smac is released into the cytosol and triggers the activity of caspases such as caspase- 3 and caspase- 9 by neutralizing inhibitor of apoptosis proteins (IAPs), especially the X-linked inhibitor of apoptosis protein (xIAP) [26]. Thus, Smac and Smac mimetics could be used as cancer therapeutic targets or tools to promote apoptosis, block pro-survival signaling, and initiate cell death in cancer cells. Many researchers have suggested that Smac and Smac mimetics could sensitize chemotherapy- or radiotherapy-resistant cancer cells [27-29]. In triple-negative breast cancer cells, Smac mimetic BV6 distinctly sensitizes resistance to paclitaxel via targeting of apoptotic pathways [28]. Henlgans et al. have shown that the Smac mimetic BV6 can sensitize colorectal cancer cells to IR by enhancing apoptosis [27]. In lung cancer A549 cells, Smac has also been reported to promote cisplatin-induced apoptosis by activating caspase-3 and caspase-9 [29].

In the present study, we first observed that the expression of SirT1 was negatively correlated with radiation sensitivity in lung cancer cell lines A549 and H460. Based on this observation, we then investigated whether SirT1 modulation could alter the radiationinduced DNA damage repair response and apoptosis to see whether the radiosensitivity of these cancer cells could be changed by SirT1 modulation. Furthermore, the molecular mechanism responsible for this alternation was illuminated.

\section{Materials and Methods}

Cell lines and cell culture conditions

Human lung cancer cell lines A549 and H460 were both obtained from the American Type Culture Collection (Manassas, VA, USA). The cells were cultured in complete high-glucose Dulbecco's modified Eagle's medium with 10\% fetal bovine serum (FBS) and 1\% penicillin/streptomycin. Both cell lines were 


\section{Cellular Physiology Cell Physiol Biochem 2018;48:304-316 and Biochemistry Published online: July 16, $2018 \quad$\begin{tabular}{l|l} 
DOI: 2018 The Author(s). Published by S. Karger AG, Basel \\
www.karger.com/cpb
\end{tabular}}

Ji et al.: Sirt1/ NF-кM/Smac Pathway for Radiation Sensitization

cultured at $37^{\circ} \mathrm{C}$ in a humidified atmosphere with $5 \% \mathrm{CO}_{2}$. When cell confluency reached $90 \%$, cells were passaged. The cell medium was changed every 2 days.

\section{Reagents and IR}

Resveratrol (RSV; 3, 5,4'-trihydroxy-trans-stilbene) and BAY11-7082 (BAY) were obtained from Sigma Chemicals (St. Louis, MO, USA). Resveratrol was prepared as a 50 -mM solution in dimethyl sulfoxide (DMSO), while BAY was prepared as a 20-mM solution in DMSO. When needed, cells were exposed to IR in a Cammacell-40 ${ }^{137}$ Cesium $\gamma$ irradiator (Atomic Energy of Canadian Inc., Mississauga, Canada) at a rate of $0.98 \mathrm{~Gy} / \mathrm{min}$.

\section{Cell survival curve and colony formation assay}

To create a cell survival curve, cells were seeded into six-well culture plates at $1 \times 10^{3}$ cells/well. After adherence for $12 \mathrm{~h}$, cells were treated with or without $50 \mu \mathrm{M}$ resveratrol, with or without $20 \mu \mathrm{M}$ BAY, or with $2 \mathrm{nM}$ siSirT1 or scrambled RNA for $48 \mathrm{~h}$ followed by $0,2,4$, or 6 Gy irradiation or sham-irradiation treatments, respectively. Immediately after irradiation, cells were cultured in fresh medium for 15 days. When plotting the cell survival curve, the mean number of colonies per well was obtained after 15 days of culture from triplicate samples. The data were plotted on a log-linear scale. For the colony formation assay, the cells were washed twice using $0.01 \mathrm{M}$ phosphate-buffered saline (PBS) after 15 days of culture, then fixed with $4 \%$ paraformaldehyde solution in $0.01 \mathrm{M}$ PBS and stained with crystal violet. Colonies containing more than 50 cells were identified as survivors under a stereomicroscope.

\section{Immunofluorescence microscopy}

DNA double-strand breaks (DSBs) formation can be detected by immunofluorescence microscopy of $\gamma \mathrm{H} 2 \mathrm{AX}$ foci [30]. Immunofluorescence microscopy was conducted as described previously with minor modifications [30, 31]. Briefly, control cells and cells treated with siSirT1 or with resveratrol for $24 \mathrm{~h}$ were collected using trypsin disaggregation. After disaggregation, the cells were seeded onto 12-well tissue culture plates, with each well enclosed by a glass cover slip, and cultured in culture medium containing $10 \%$ FBS at $37^{\circ} \mathrm{C}$ for $12 \mathrm{~h}$. Then, the cultures were treated with or without $4 \mathrm{~Gy}$ irradiation and then cultured for another $6 \mathrm{~h}$. Cells were washed with PBS, fixed in 4\% paraformaldehyde for $15 \mathrm{~min}$, washed three times with PBS, and permeabilized with $0.3 \%$ Triton X-100 for $15 \mathrm{~min}$ at room temperature. Subsequently, cells were washed with PBS and blocked with $1 \%$ bovine serum albumin for $1 \mathrm{~h}$ at room temperature and incubated with a primary antibody overnight at $4^{\circ}$ C. After three washes with PBS, cells were incubated in the dark with fluorescein-conjugated secondary antibodies for $1 \mathrm{~h}$. Nuclei were stained with DAPI (4',6-diamidino2-phenylindole dihydrochloride) for $10 \mathrm{~min}$. Thereafter, cells were visualized using an EVOS inverted fluorescence microscope (Nikon, Tokyo, Japan). To prevent bias in selection of cells that display foci, over 200 randomly selected cells from each treatment group were counted. Cells with ten or more foci of any size were classified as positive. All experiments were repeated in triplicate.

\section{Annexin V/PI flow cytometric analysis}

The percentage of cells actively undergoing apoptosis was quantified by means of a combined staining with annexin and propidium iodide using the Annexin V-FITC Apoptosis Detection Kit (Baosai Biotech, Shanghai, China) according to the manufacturer's instructions. Briefly, following treatment with resveratrol or siSirT1 or BAY for $48 \mathrm{~h}$, cells were harvested and analyzed by using MRFLOW software (Mindray, Shenzhen, China). To study the combined effect of IR with resveratrol or siSirT1 or BAY, cells were treated with resveratrol or BAY or transfected with siRNA for $24 \mathrm{~h}$ and then treated with IR ( $4 \mathrm{~Gy}$ ) for $24 \mathrm{~h}$ before apoptosis analysis.

\section{RNA interference}

SirT1 siRNA was used to knock down SirT1 in A549 cells and H460 cells to explore whether the inhibition of SirT1 can affect apoptosis or radiosensitivity. Specific SirT1 siRNAs and the control mismatch oligonucleotides were synthesized by GenePharma (Suzhou, China). RNA interference assays were performed as described before [32]. Briefly, A549 cells and H460 cells were transfected with $2 \mathrm{nM}$ siRNA using Lipofectamine RNAi Max (Thermo Fisher Scientific, Waltham, MA, USA) and OptiMEM (Thermo Fisher Scientific). Then, cells were further cultured for $24 \mathrm{~h}$ for transfection and gene knockdown. Knockdown cells 


\section{Cellular Physiology Cell Physiol Biochem 2018;48:304-316 \begin{tabular}{c|c} 
DOI: 10.1159/000491730 & Ond Biochemistry \\
Published online: July 16, 2018 & $\begin{array}{l}\text { 2018 The Author(s). Published by S. Karger AG, Basel } \\
\text { www.karger.com/cpb }\end{array}$ \\
\cline { 2 - 3 }
\end{tabular} Ji et al.: Sirt1/ NF-кM/Smac Pathway for Radiation Sensitization}

were treated with or without 4 Gy radiation. Cells were further subjected to apoptosis detection, protein extraction, and western blotting.

Protein extraction and western blotting

A549 and H460 cells were collected, washed twice with 0.01 M PBS, and lysed on ice with RIPA cell lysis reagent (Beyotime, Nanjing, China) for $30 \mathrm{~min}$. Cells were centrifuged at 12, $000 \mathrm{rpm}$ for $15 \mathrm{~min}$ at $4^{\circ} \mathrm{C}$ to remove insoluble proteins. Supernatants were collected and protein concentrations were determined using a BCA protein Assay Kit (Beyotime) with a Nanodrop Spectrophotometer (Thermo Fisher Scientific). Fifty-microgram samples of total protein extracts were separated using $10 \%$ sodium dodecyl sulfatepolyacrylamide gel electrophoresis and transferred onto polyvinylidene difluoride membranes. After being blocked with $5 \%$ milk solution, membranes were washed three times with Tris-buffered saline-tween (TBST) solution. Then, membranes were probed overnight at $4^{\circ} \mathrm{C}$ with primary antibodies against SirT1, Smac, NF-кB, caspase-3, cleaved caspase-3, caspase-9, cleaved caspase-9, PARP, cleaved PARP, and GAPDH. After being washed with TBST, the membranes were incubated with goat anti-rabbit or goat anti-mouse secondary antibodies conjugated with horseradish peroxidase for $3 \mathrm{~h}$ at room temperature. Membranes were processed for protein detection using the ECL Chemiluminescence Detection Kit (Proteintech, Chicago, IL, USA). Changes in protein expression were quantified using Quantity One Software (Bio-Rad, Hercules, CA, USA) and presented in graphical format.

\section{Statistical analysis}

The results are represented as means \pm standard deviations and are statistically compared with the untreated control group or compared within treated groups using Student's $t$-tests as implemented by SPSS 19.0 software (IBM Corp, Armonk, NY, USA). A threshold of $p<0.05$ was used to determine statistical significance.

\section{Results}

SirT1 is associated with the radiation sensitization of lung cancer cells

In order to study the relationship between SirT1 and radiosensitivity, two common lung cancer cell lines with different radiation sensitivities, namely, A549 and H460, were used in this study. First, we performed a colony formation assay under different doses of irradiation to estimate the cell viability of the two cell lines. As shown in the clonogenic survival curve (Fig. 1A), H460 cells are more sensitive to radiation compared with A549 cells, with IC50 values (as analyzed by SPSS 19.0) of 2.69 Gy and 1.92 Gy for A549 and H460, respectively. Interestingly, the basal expression level of SirT1 in A549 cells was observed to be obviously much higher than that in H460 cells (Fig. 1B). These results indicate that the expression of SirT1 may be negatively correlated with radiosensitivity for lung cancer cell lines. To provide further evidence of the relevance of SirT1 to radiosensitivity in lung cancer cell lines, the radiosensitivity of the two cell lines was tested under SirT1 knockdown or activation conditions. As illustrated in Fig. 1C and Fig. 1E, the proliferation of both A549 cells and H460 cells was significantly inhibited when the expression of SirT1 was knocked down. In addition, siSirT1 enhanced the radiosensitivity of lung cancer cell lines, especially in the A549 cell line. When SirT1 was activated by resveratrol, the radiosensitivity of A549 cells and H460 cells were both significantly reduced (Fig. 1D and Fig. 1F).

SirT1 is associated with radiation-induced DNA damage in lung cancer cells

DNA DSB damage is the most important effect of IR. Phosphorylation of histone H2AX at Ser15 $(\gamma \mathrm{H} 2 \mathrm{AX})$ is one of the initial steps of the DNA damage response upon occurrence of a DSB, spanning several $\mathrm{kb}$ around the DSB, and can be visualized as discrete foci [30]. We thus performed immunofluorescent staining of $\gamma \mathrm{H} 2 \mathrm{AX}$ to measure the influence of SirT1 expression on dsDNA break formation in A549 and $\mathrm{H} 460$ cells following $\gamma$ irradiation. As shown in Fig. 2 and Fig. 2A in particular, the radiated A549 cells had an increase in the $\gamma \mathrm{H} 2 \mathrm{AX}$ 
foci, and the $\gamma \mathrm{H} 2 \mathrm{AX}$-foci-positive rate increased to $38.2 \%$ compared with $0 \%$ in the nonirradiation control group. When pretreated with siSirT1 and then treated with irradiation, siSirT1-mediated SirT1 depletion distinctly increased the $\gamma \mathrm{H} 2 \mathrm{AX}$-foci-positive rate from $38.2 \%$ to $73.3 \%$ compared with the group that received irradiation alone (Fig. 2A and Fig. 2B). In contrast, when SirT1 was activated with resveratrol, the degree of DNA damage induced by radiation was significantly reduced (Fig. 2A), and the $\gamma \mathrm{H} 2 \mathrm{AX}$-foci-positive rate was significantly decreased compared with the irradiation alone group (Fig. 2B). Similar results were also observed in H460 cells (Fig. 2C).

\section{Modulation of SirT1 regulates radiation-induced apoptosis in lung cancer cells}

To investigate the impact of the regulation of SirT1 on radiation sensitivity in the A549 and $\mathrm{H} 460$ cells, apoptosis of cells was examined by combined staining with Annexin $\mathrm{V}$ and PI. As shown in Fig. 3, in the siSirT1 combined treatment group, the apoptotic rates were approximately $26.7 \%$ in A549 cells and $31.2 \%$ in $\mathrm{H} 460$ cells, in contrast to approximately $8.9 \%$ and $19.3 \%$ in the radiation alone group. However, when pretreated with the SirT1 activator resveratrol, the apoptotic rates were markedly decreased (approximately $5.7 \%$ and 8.2\%) both in A549 and H460 cells, in contrast to the radiation alone group. Consistent with the observation of apoptosis, we also detected the activated cleavage products of caspase-3, caspase-9, and PRAP by western blot. As shown in Fig. 4A and 4C, the activated cleavage products of caspase-3, caspase-9, and PRAP were identified in siSirT1-treated A549 cells. Trace amounts of activation cleavage were detectable in the irradiated alone group, while little was detectable in the controls. However, when pretreated with resveratrol, the activated cleavage products of caspase-3, caspase- 9 , and PRAP were not obviously changed compared with the irradiated alone group for A549 cells (Fig. 4A and 4D). This was in contrast to the A549 cell line, which when pretreated with siSirT1 in H460 cells, showed no obvious increase in activated cleavage products of caspase-3, caspase-9, and PRAP compared with the irradiated alone group (Fig. 4B and 4E). Unexpectedly, the activation cleavage products were significantly increased in the irradiated alone $\mathrm{H} 460$ cells compared with the control group and were remarkably reduced by resveratrol pretreatment combined with irradiation in H460 cells compared with the irradiated alone group (Fig 4B and 4F).

Fig. 1. SirT1 is negatively associated with radiation sensitization in lung cancer cells. (A) Colony formation assay. Cell surviving fraction was measured after different doses of radiation treatment of A549 and H460 cell lines. (B) The expression of SirT1 was measured in A549 and H460 cell lines. (C) Effect of SirT1 knockdown on radiation sensitivity in A549 and H460 cell lines. (D) Effects of SirT1 activation on cellular radiosensitivity in A549 and H460 cell lines after resveratrol treatment. (E) Effect of SirT1 knockdown on cell survival after radiation treatment of A549 and H460 cell lines. (F) Effect of SirT1 activation on cell survival after radiation treatment of $\mathrm{A} 549$ and $\mathrm{H} 460$ cell lines. Radiation doses of 2, 4, and 6 Gy were used in these experiments. The numbers of colonies per well were noted after a 15-day culture. The results presented are the mean \pm SD of three experiments, each conducted in triplicate.

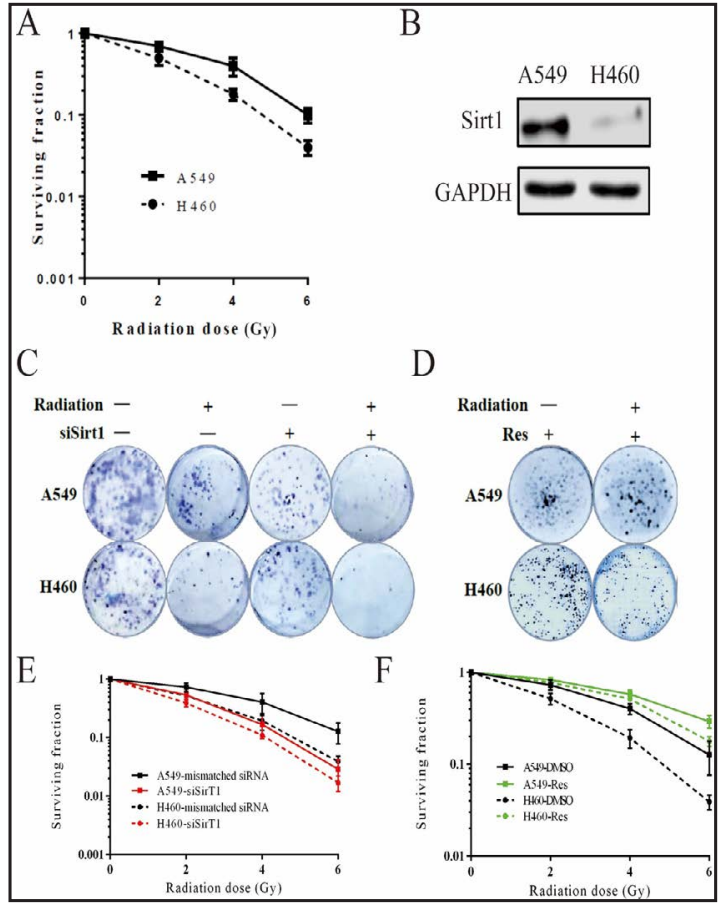




\section{Cellular Physiology and Biochemistry

Fig. 2. The expression of SirT1 affected ionizing radiation (IR)-induced DNA doublestrand breaks (DSBs) in A549 cells. DSB levels were detected by immunofluorescence staining of $\gamma \mathrm{H} 2 \mathrm{AX}$ foci (green dots). (A) Representative micrographs. (B) $\gamma \mathrm{H} 2 \mathrm{AX}$ positive rate (\%) for the A549 cell line. (C) $\gamma \mathrm{H} 2 \mathrm{AX}$ positive rate $(\%)$ for the $\mathrm{H} 460$ cell line. A radiation dose of 4 Gy was used in these experiments. Cells were cultured for another $6 \mathrm{~h}$ after irradiation, and then subjected to an immunofluorescence staining analysis of $\gamma \mathrm{H} 2 \mathrm{AX}$ foci. Results are expressed as mean \pm $\mathrm{SD}(\mathrm{n}=4){ }^{* *} \mathrm{p}<0.05$.

Fig. 3. Apoptosis levels of A549 and H460 cell lines can be regulated by the expression of SirT1.The apoptotic rates were assayed by an Annexin V/PI flow cytometric analysis. A radiation dose of 4 Gy was used in these experiments. Cells were cultured for another $24 \mathrm{~h}$ after radiation exposure, and then subjected to an Annexin V/PI flow cytometric analysis. Results are expressed as mean \pm SD $(\mathrm{n}=4) .{ }^{* *} \mathrm{p}<0.05$; $^{* * *} \mathrm{p}<0.01$.
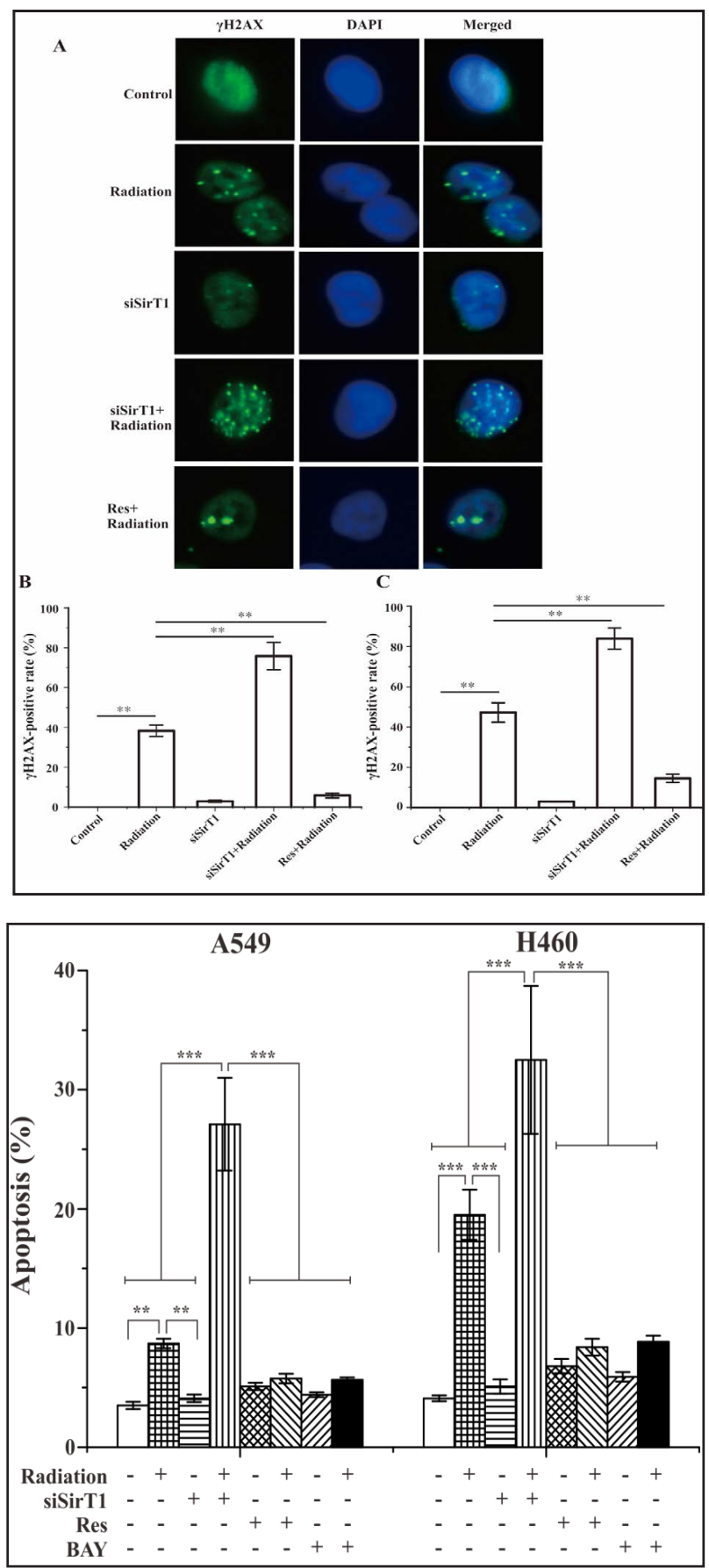

Modulation of SirT1 regulates the expression of Smac in lung cancer cells

To explore the molecular mechanisms of SirT1-regulated cell apoptosis, we analyzed the effect of SirT1 on Smac in A549 and H460 cells. The expression of SirT1 and Smac in A549 and $\mathrm{H} 460$ cells was analyzed by western blotting. The results suggested that SirT1 was highly expressed in A549 cells and Smac was highly expressed in H460 cells (Fig. 5). Treatment with siSirT1 resulted in a significant reduction of SirT1 in both A549 and H460 cells and a significant induction of Smac in A549 cells (Fig. 5A and 5C). However, the expression of SirT1 was obviously activated, and the expression of Smac was remarkably reduced in both A549 and H460 cells when treated with the SirT1 activator resveratrol (Fig. 5B and 5D). These 


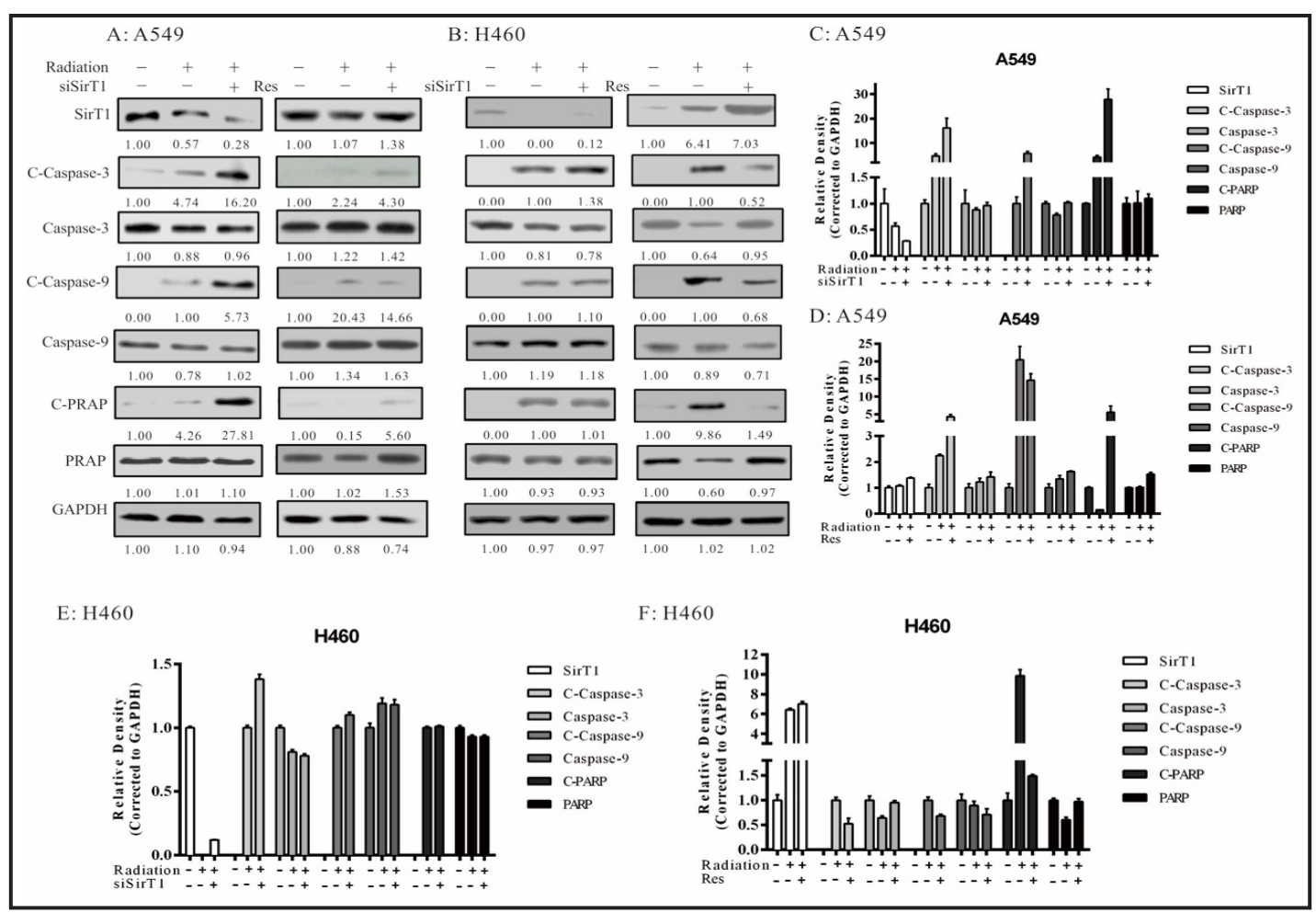

Fig. 4. Effects of SirT1 on the expression of apoptosis-related proteins in whole protein extracts from (A) A549 and (B) H460 cell lines. Quantization of SirT1 and apoptosis-related proteins levels in whole protein extracts in A549 cell lines when treated with (C) siSirT1 and radiation or (D) Res and radiation. Quantization of SirT1 and apoptosis-related proteins levels in whole protein extracts in $\mathrm{H} 460$ cell lines when treated with (E) siSirT1 and radiation or(F) Res and radiation. A radiation dose of 4 Gy was used in these experiments. Cells were cultured for another $24 \mathrm{~h}$ after radiation exposure and then used for a western blot analysis. This experiment was repeated three times, each time in triplicate.

results demonstrate that the expression of Smac was negatively regulated by SirT1 in both A549 and H460 cells.

$N F-\kappa B$ is a critical SirT1-regulated mediator of Smac in lung cancer cells

The nuclear factor- $\kappa \mathrm{B}$ family has been reported as one of the substrates of SirT1 and to be associated with cell apoptosis. Accordingly, we examined the relationship between the expression of NF- $\mathrm{KB}$ and SirT1 in A549 and H460 cells. As shown in Fig. 6, after treatment with siSirT1, the expression of SirT1 was remarkably depleted both in A549 and H460 cells (Fig. 6A, B, E and G), while the expression of NF- $\kappa \mathrm{B}$ and Smac were significantly increased in A549 cells (Fig. 6A and 6E). Treatment with resveratrol resulted in an induction of SirT1 expression, but a reduction of NF- $\kappa \mathrm{B}$ and Smac expression in both A549 and H460 cells (Fig. $6 \mathrm{~A}, \mathrm{~B}, \mathrm{~F}$ and $\mathrm{H}$ ). These results suggest that NF- $\mathrm{KB}$ is similar to Smac and could be negatively regulated by SirT1 in both A549 and H460 cells. Based on the above results, we further explored whether NF- $\kappa B$ is a critical SirT1-regulated mediator of Smac in A549 and H460 cells. To this end, we applied BAY, an inhibitor of NF- $\kappa \mathrm{B}$ to downregulate the expression of NF- $\mathrm{KB}$ and observe the apoptosis of cells and expression of Smac. As we expected, BAY significantly inhibited the expression of NF- $\mathrm{BB}$ both in A549 and H460 cells (Fig 6 C, I and J). Compared with the radiation alone group, the apoptosis rate of the BAY and irradiation co-treatment group was decreased in both A549 and H460 cell lines (Fig. 3). Meanwhile, the expression of Smac was suppressed when cells were treated with BAY. These results strongly indicate that Smac is transcriptionally regulated by the nuclear factor- $\kappa \mathrm{B}$ family. 
Fig. 5. Effects of SirT1 on the expression of Smac in whole protein extracts from A549 and H460 cell lines. (A) The expression of Smac was activated when the expression of SirT1 was suppressed by siSirT1. (B) The expression of Smac was inhibited when the expression of SirT1 was induced by resveratrol. (C) Quantization of SirT1 and Smac levels in A549 and $\mathrm{H} 460$ cell lines when the expression of SirT1 was suppressed by siSirT1. (D) Quantization of SirT1 and Smac levels in A549 and $\mathrm{H} 460$ cell lines when the expression of SirT1 was induced by resveratrol. A radiation dose of 4 Gy was used in these experiments. Cells were cultured for another $24 \mathrm{~h}$ after radiation exposure and then used for western blot analysis. This experiment was repeated three times, each time in triplicate.

Fig. 6. Effects of SirT1 on the expression of NF- $\kappa B$ and Smac in whole protein extracts from (A) A549 and (B) H460 cell lines. Effects of NF- $\mathrm{kB}$ on the expression of Smac in whole protein extracts from (C) A549 and (D) H460 cell lines. Quantization of SirT1, NF-кB, and Smac levels in whole protein extract in A549 cell lines when treated with (E) siSirT1 and radiation, (F) Res and radiation, or (I) BAY and radiation. Quantization of SirT1, NF-кB, and Smac levels in whole protein extract in $\mathrm{H} 460$ cell lines when treated with $(G)$ siSirT1 and radiation, $(\mathrm{H})$ Res and radiation, or $(\mathrm{J})$ $\mathrm{BAY}$ and radiation. A radiation dose of $4 \mathrm{~Gy}$ was used in these experiments. Cells were cultured for another $24 \mathrm{~h}$ after radiation exposure and then used for western blot analysis. This experiment was repeated three times, each time in triplicate.
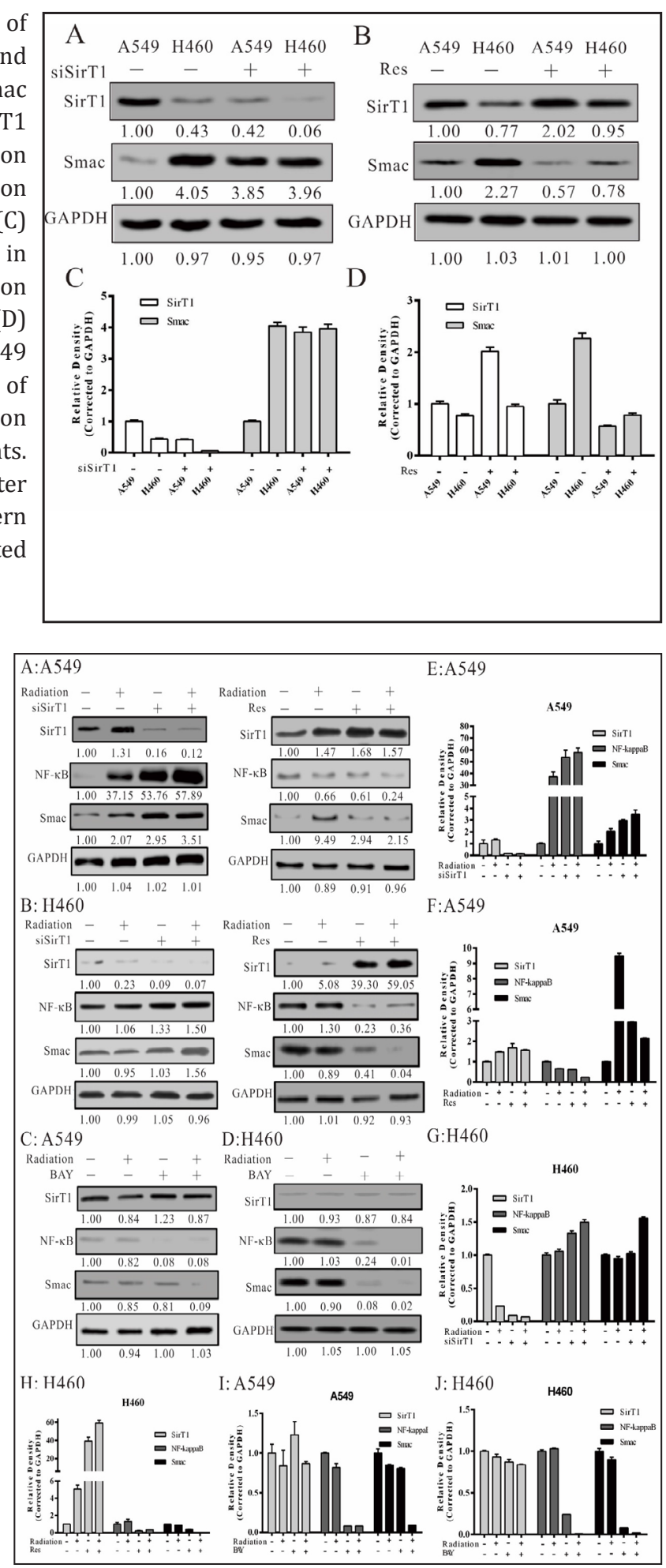

\section{Discussion}

SirT1, a member of the sirtuin family, plays an important role in the regulation of stress responses and cell fate determination in mammalian cells. Recent works have also indicated that SirT1 is overexpressed in several cancer cells including lung cancer cells, and the 
expression of SirT1 could be associated with resistance to chemotherapy and radiotherapy through its interactions with FOXO, Bcl-2, p-21, p-53, AKT, and ataxia telangiectasia mutated (ATM) [21, 23]. In lung cancer cell line A549, the downregulation of SirT1 can induce apoptosis and enhance radiation sensitization [8]. However, the underlying mechanism for this phenomenon has remained unclear. In the present study, we, for the first time, demonstrate that SirT1 regulates the apoptosis and radiation sensitivity of lung cancer cell lines A549 and H460 through the SirT1/NF- $\mathrm{BB} /$ Smac pathway. Based on other published findings and our results, the SirT1/NF- $\mathrm{\kappa B} / \mathrm{Smac}$ pathway may be a new potential target pathway in therapy for non-small cell lung carcinoma (NSCLC).

Lung cancer remains a leading cause of cancer-related deaths worldwide [33]. NSCLC, which can be divided into lung cell adenocarcinoma, squamous lung cell carcinoma, and large lung cell carcinoma, accounts for $75 \%$ of lung cancer cases [34]. Although belonging to NSCLC, A549 and H460 cells represent lung adenocarcinoma and large lung cell carcinoma, respectively. $\mathrm{H} 460$ cells have been reported to be more susceptible to radiation compared with A549 cells [34-36]. In addition, studies have also shown that H460 cells were more susceptible to apoptosis compared with A549 cells [37]. In this study, firstly, we demonstrated that the two lung cancer cell lines A549 and $\mathrm{H} 460$ have different radiosensitivities. A549 cells were more resistant to radiation compared with $\mathrm{H} 460$ cells, and this result was consistent with previous research results (Fig. 1A). Subsequently, the expression levels of SirT1 in both cell lines were analyzed. This result suggested that the expression level of SirT1 is higher in A549 cells with less radiosensitivity than that in H460 cells (Fig. 1B). When treated with siSirT1, the radiation sensitivity of both cell lines increased, though the observation could be reversed when treated with SirT1 agonists of resveratrol (Fig. 1C, D, E and F). Taken together, these results indicate that there is a negative correlation between the expression level of SirT1 and the radiosensitivity of the two lung cancer cell lines A549 and H460.

It is well documented that IR causes an increase in intracellular reactive oxygen species production, thereby resulting in oxidative damage to proteins, lipids, and DNA, which subsequently leads to DNA DSBs $[38,39]$. In this study, we show that radiation leads to elevated levels of DNA damage in both A549 and H460 cells, with further increases occurring when pretreated with siSirT1 (Fig. 2). However, the levels of DNA damage were alleviated when cells were pretreated with resveratrol before irradiation, for both A549 and H460 cells (Fig 2). These data demonstrated that SirT1 may regulate the radiosensitivity of lung cancer cells by influencing DNA damage repair capabilities. Dobbin et al. suggested that SirT1 can maintain genomic stability by stimulating autophosphorylation of ATM and deacetylation of histone deacetylase HDAC1 to induce DNA damage repair processes in neurons [23]. Another study also showed that impaired DNA damage response and genome instability are detected in SirT1 mutant mice [21]. Given these conclusions, our current results can be interpreted as indicating that SirT1 affects the radiation sensitivity of cells by affecting the DNA damage repair process.

DNA damages are highly deleterious because they can be lethal if they remain unrepaired or are mis-repaired [40]. To cope with these lesions, cells develop multiple interaction pathways, referred to as the DNA damage response, which leads either to damage repair or to apoptosis depending on the extent of the damage [41]. Given the observation of an increase in DNA damage to siSirT1-treated cells, we investigated whether the levels of DNA damage could lead to cell apoptosis. In the present study, our data suggested that the percentage of apoptotic cells was significantly increased when siRNA was used to interfere with SirT1 expression (Fig. 3). Meanwhile, when resveratrol was used, the percentage of apoptotic cells was decreased (Fig. 3). In addition, as shown in Fig. 3, when treated with radiation alone, the apoptotic rate of A549 cells was approximately 8.9\%, while the apoptotic rate of $\mathrm{H} 460$ cells was as high as $19.3 \%$. This high apoptotic rate in $\mathrm{H} 460$ cells may be owing to their weak expression of SirT1. When compared with the radiation alone group, the apoptotic rate of A549 cells treated with siSirt1 combined with radiation was increased by $17.8 \%$, and this may be owing to the significant knockdown of SirT1 expression. However, in H460 cells, this result was increased only by $11.2 \%$, which may be owing to the weak expression of SirT1 


\section{Cellular Physiology Cell Physiol Biochem 2018;48:304-316 \begin{tabular}{l|l} 
and Biochemistry Published online: July 16, 2018 & $\begin{array}{l}\text { ( ) } 2018 \text { The Author(s). Published by S. Karger AG, Basel } \\
\text { www.karger.com/cpb }\end{array}$
\end{tabular}}

Ji et al.: Sirt1/ NF-кM/Smac Pathway for Radiation Sensitization

and the relatively weak knockdown effect. Caspases, a family of cysteine proteases that are highly conserved in multicellular organisms, function as central regulators of apoptosis [42]. In addition, poly (ADP-ribose) polymerase (PARP) also plays key signaling roles in apoptosis. Therefore, we next detected the expression level of caspase- 3 , caspase- 9 , PARP as well as cleaved-caspase-3, caspase-9, and PARP. As shown in Fig. 4, in the combined siSirT1 and irradiation treatment group, the expression of SirT1 was suppressed and the expression levels of cleaved caspase-3, caspase- 9 and PARP were remarkably increased, in both lung cancer cells lines. Additionally, the expression levels of caspase-3, caspase-9, and PARP were decreased to a certain extent (Fig. 4). In contrast, in the resveratrol and irradiation combined treatment group, the expression levels of activated cleaved caspase-3, caspase- 9 and PARP were obviously decreased in H460 cells compared with the irradiation alone treatment group (Fig. 4). Notably, the obvious decrease was not detected in A549 cells under the same conditions (Fig. 4). This may be associated with the lower expression levels of cleaved caspase 3, caspase-9, and PAPR in A549 cells under irradiation alone conditions. Addtionally, we suggest that the decreased expression of cleaved caspase 3, caspase- 9 and PARP in A549 cells resulted in lower apoptosis and stronger radioresistance under irradiation conditions when compared with H460 cells. Similar results have been obtained in multiple cell lines, including gliomas, colon cancer cells and lung cancer cell line A549 [8, 43, 44]. Consistent with previous reports, our experiments demonstrated that SirT1 can regulate apoptosis in lung cancer cell lines.

Furthermore, we aimed to identify which effectors and pathways are used by SirT1 to regulate apoptosis. Smac, an important pro-apoptotic protein in mitochondria-driven apoptosis, is released into the cytosol and triggers caspase activation [25]. Therefore, we analyzed the relationship between SirT1 and Smac. As shown in Fig. 5A, the expression of Smac was induced when the expression of SirT1 was downregulated by siSirT1, especially in A549 cells. However, the expression of Smac was suppressed when SirT1 was activated by resveratrol, especially in $\mathrm{H} 460$ cells. Our data suggest that SirT1 negatively regulates the expression of Smac in both A549 and H460 cells. It is worth noting that knocking down or activating SirT1 in A549 and H460 cells has different effects on the expression of apoptosisrelated protein (Fig. 4) and Smac (Fig. 5). These different responses may be caused by the high expression of SirT1 in A549 cells, such that SirT1 knockdown has a greater effect on the expression of related proteins in these cells. However, in H460 cells, SirT1 expression is low, and the activation of SirT1 thus has a greater effect on the expression of related proteins.

Previous studies have indicated that NF- $\kappa B$ is one of the downstream substrates of SirT1 because the RelA/p65 subunit of NF- $\kappa$ B can be regulated by SirT1 [45]. NF- $\kappa B$, in most reports, functions to protect cells from death through anti-apoptotic signals [46]. However, $\mathrm{NF}-\kappa \mathrm{B}$ has also been reported to contribute to apoptosis [47]. Accordingly, we hypothesized that NF- $\mathrm{KB}$ is a critical mediator of SirT1-induced apoptosis. In both A549 and H460 cells, we observed that the expression of NF- $\kappa$ B was negatively regulated by SirT1 (Fig. 6A, B, E-H). As is known, SirT1, which belongs to HDAC group III, can deacetylate the lysine residues on the N-terminal tails of the core histone proteins, resulting in coiling of the DNA and subsequently decreased transcription $[19,48]$. As such, we speculated that SirT1 reduced the expression of NF- $\kappa \mathrm{B}$ by deacetylation of histones and thus protected cells from the proapoptotic effect of NF- $\kappa$ B. Additionally, Smac or Smac mimetics have also been reported to sensitize cells to apoptosis in a NF- $\kappa B$-dependent manner $[49,50]$. When the expression of NF- $\kappa B$ was suppressed by the inhibitor BAY, the expression of Smac was significantly reduced, while SirT1 was not affected in both A549 and H460 cells (Fig. 6C, D, I and J). As such, we speculate that the deacetylation of histones by SirT1 affects the expression of NF- $\kappa B$, which in turn affects the expression of Smac. In addition, SirT1 deacetylase can also regulate NF$\kappa B$ transcription through direct interactions with NF- $\kappa B$ subunit RelA/p65 [51]. This may then reduce the expression of Smac. Based on the above results, our data confirmed that SirT1 regulates apoptosis through the SirT1-NF-kB-Smac signaling pathway in lung cancer cell lines A549 and H460. 


\section{Cellular Physiology Cell Physiol Biochem 2018;48:304-316 \begin{tabular}{l|l} 
and Biochemistry Published 10.1159/000491730 & $\begin{array}{l}\text { D) } 2018 \text { The Author(s). Published by S. Karger AG, Basel } \\
\text { www.karger.com/cpb }\end{array}$ \\
\hline
\end{tabular} Ji et al.: Sirt1/ NF-кM/Smac Pathway for Radiation Sensitization}

Taken together, our study for the first time shows that SirT1 regulates the apoptosis and radiosensitivity in lung cancer cell lines A549 and H460 via the SirT1-NF-kB-Smac signaling pathway. Our results also highlight the potential of these novel targets in the treatment of lung cancer. However, it should be noted that the regulatory mechanism of SirT1 with respect to apoptosis and radiosensitivity in lung cell lines should be further elucidated in vivo.

\section{Acknowledgements}

This study was supported by the National Natural Science Foundation of China (31670859), the CAMS Innovation Fund for Medical Science (2017-I2M-1-016), the Natural Science Foundation of Tianjin (15KPXM01SF056), the Non-profit Central Research Institute Fund of Chinese Academy of Medical Sciences (2016ZX310068, 2016ZX310198, 2016RC310019, 2016RC310017), the PUMC Youth Fund and the Fundamental Research Funds for the Central Universities (3332016100, 10023201601602), and Research Funds for the Innovation Team of IRM-CAMS (1650).

\section{Disclosure Statement}

These authors have no competing interests to disclose.

\section{References}

1 Huang N, Zhu J, Liu D, Li YL, Chen BJ, He YQ, Liu K, Mo XM, Li WM: Overexpression of Bcl-2-associated death inhibits A549 cell growth in vitro and in vivo. Cancer Biother Radio 2012;27:164-168.

-2 Rizvi NA, Mazières J, Planchard D, Stinchcombe TE, Dy GK, Antonia SJ, Horn L, Lena H, Minenza E, Mennecier B, Otterson GA, Campos LT, Gandara DR, Levy BP, Nair SG, Zalcman G, Wolf J, Souquet P-J, Baldini E, Cappuzzo F, Chouaid C, Dowlati A, Sanborn R, Lopez-Chavez A, Grohe C, Huber RM, Harbison CT, Baudelet C, Lestini BJ, Ramalingam SS: Activity and safety of nivolumab, an anti-PD-1 immune checkpoint inhibitor, for patients with advanced, refractory squamous non-small-cell lung cancer (CheckMate 063): a phase 2, single-arm trial. Lancet oncol 2015;16:257-265.

- Hanna N, Neubauer M, Yiannoutsos C, McGarry R, Arseneau J, Ansari R, Reynolds C, Govindan R, Melnyk A, Fisher W, Richards D, Bruetman D, Anderson T, Chowhan N, Nattam S, Mantravadi P, Johnson C, Breen T, White A, Einhorn L, Hoosier Oncology G, Oncology US: Phase III study of cisplatin, etoposide, and concurrent chest radiation with or without consolidation docetaxel in patients with inoperable stage III non-small-cell lung cancer: the Hoosier Oncology Group and U.S. Oncology. J Clin Oncol 2008;26:57555760.

4 Sensebé L, Bourin P: Mesenchymal stem cells for therapeutic purposes. Transplantation 2009;87:49-53.

5 Ye Z, Fang B, Pan J, Zhang N, Huang J, Xie C, Lou T, Cao Z: miR-138 suppresses the proliferation, metastasis and autophagy of non-small cell lung cancer by targeting Sirt1. Oncol Rep 2017;37:3244-3252.

6 You S, Li R, Park D, Xie M, Sica GL, Cao Y, Xiao ZQ Deng X: Disruption of STAT3 by niclosamide reverses radioresistance of human lung cancer. Mol Cancer Ther 2014;13:606-616.

7 Feng H, Wang J, Xu J, Xie C, Gao F, Li Z: The expression of SIRT1 regulates the metastaticplasticity of chondrosarcoma cells by inducing epithelial-mesenchymal transition. Sci Rep 2017;7:41203.

8 Sun Y, Sun D, Li F, Tian L, Li C, Li L, Lin R, Wang S: Downregulation of Sirt1 by antisense oligonucleotides induces apoptosis and enhances radiation sensitization in A549 lung cancer cells. Lung Cancer 2007;58:2129.

-9ee JW KS, Kotliarov Y, Li AG, Su Q Nicholas MD, Pastorino S, Benjimin WP, Christopher N, Zhang W, Park JK, Fine HA: Tumor stem cells derived from glioblastomas cultured in bEGF and EGF more closely mirror the phenotype and genotype of primary tumors than do serum-cultured cell lines. Cancer Cell 2006;9:391-403.

10 Saunders LR, Verdin E: Sirtuins: critical regulators at the crossroads between cancer and aging. Oncogene 2007;26:5489-5504. 


\section{Cellular Physiology Cell Physiol Biochem 2018;48:304-316

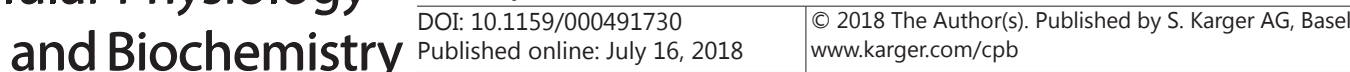

Ji et al.: Sirt1/ NF-кM/Smac Pathway for Radiation Sensitization

11 Singh P, Hanson PS, Morris CM: SIRT1 ameliorates oxidative stress induced neural cell death and is downregulated in Parkinson's disease. BMC Neurosci 2017;18:46.

12 L G: Linking DNA Damage, NAD(+)/SIRT1, and Aging. Cell Metab 2014;20:706-707.

$\checkmark 13$ Hou M, Zuo X, Li C, Zhang Y, Teng Y: Mir-29b Regulates Oxidative Stress by Targeting SIRT1 in Ovarian Cancer Cells. Cellular Physiol Biochem 2017;43:1767.

14 Yao H, Rahman I: Perspectives on translational and therapeutic aspects of SIRT1 in inflammaging and senescence. Biochem Pharmacol 2012;84:1332-1339.

15 Finkel T, Deng CX, Mostoslavsky R: Recent progress in the biology and physiology of sirtuins. Nature 2009;460:587-591.

16 Liu ZJ, Zhuge Y, Velazquez OC: Trafficking and differentiation of mesenchymal stem cells. J Cell Biochem 2009;106:984.

17 Kumar A, Chauhan S: How much successful are the medicinal chemists in modulation of SIRT1: A critical review. Eur J Med Chem 2016;119:45-69.

18 Ray U, Roy SS, Chowdhury SR: Lysophosphatidic Acid Promotes Epithelial to Mesenchymal Transition in Ovarian Cancer Cells by Repressing SIRT1. Cellular Physiol Biochem 2017;41:795.

19 Dali-Youcef N, Lagouge M, Froelich S, Koehl C, Schoonjans K, Auwerx J: Sirtuins: the 'magnificent seven', function, metabolism and longevity. Ann Med 2007;39:335-345.

20 Huffman DM, Grizzle WE, Bamman MM, Kim JS, Eltoum IA, Elgavish A, Nagy TR: SIRT1 is significantly elevated in mouse and human prostate cancer. Cancer Res 2007;67:6612-6618.

-21 Wang RH, Sengupta K, Li C, Kim HS, Cao L, Xiao C, Kim S, Xu X, Zheng Y, Chilton B, Jia R, Zheng ZM, Appella E, Wang XW, Ried T, Deng CX: Impaired DNA damage response, genome instability, and tumorigenesis in SIRT1 mutant mice. Cancer Cell 2008;14:312-323.

-22 Peng L, Yuan Z, Li Y, Ling H, Izumi V, Fang B, Fukasawa K, Koomen J, Chen J, Seto E: Ubiquitinated sirtuin 1 (SIRT1) function is modulated during DNA damage-induced cell death and survival. J Biol Chem 2015;290:8904-8912.

23 Dobbin MM, Madabhushi R, Pan L, Chen Y, Kim D, Gao J, Ahanonu B, Pao PC, Qiu Y, Zhao Y, Tsai LH: SIRT1 collaborates with ATM and HDAC1 to maintain genomic stability in neurons. Nat Neurosci 2013;16:10081015.

24 Ling S, Li J, Shan Q, Dai H, Lu D, Wen X, Song P, Xie H, Zhou L, Liu J, Xu X, Zheng S: USP22 mediates the multidrug resistance of hepatocellular carcinoma via the SIRT1/AKT/MRP1 signaling pathway. Mol Oncol 2017;11:682-695.

25 Saita S, Nolte H, Fiedler KU, Kashkar H, Venne AS, Zahedi RP, Kruger M, Langer T: PARL mediates Smac proteolytic maturation in mitochondria to promote apoptosis. Nat Cell Biol 2017;19:318-328.

-26 Fulda S, Vucic D: Targeting IAP proteins for therapeutic intervention in cancer. Nat Rev Drug Discov 2012;11:109-124.

27 Hehlgans S, Oppermann J, Reichert S, Fulda S, Rodel C, Rodel F: The SMAC mimetic BV6 sensitizes colorectal cancer cells to ionizing radiation by interfering with DNA repair processes and enhancing apoptosis. Radiat Oncol 2015;10:198.

28 Ballarini F, Biaggi M, Ottolenghi A, Sapora O: Cellular communication and bystander effects: a critical review for modelling low-dose radiation action-mutation research. Mutat Res 2002;501:1-12.

29 Qin S, Yang C, Wang X, Xu C, Li S, Zhang B, Ren H: Overexpression of Smac promotes Cisplatin-induced apoptosis by activating caspase-3 and caspase- 9 in lung cancer A549 cells. Cancer Biother Radio 2013;28:177-182.

30 Revenco T, Lapouge G, Moers V, Brohee S, Sotiropoulou PA: Low Dose Radiation Causes Skin Cancer in Mice and Has a Differential Effect on Distinct Epidermal Stem Cells. Stem Cells 2017;35:1355-1364.

31 Zhao J, Guo Z, Pei S, Song L, Wang C, Ma J, Jin L, Ma Y, He R, Zhong J, Ma Y, Zhang H: pATM and gammaH2AX are effective radiation biomarkers in assessing the radiosensitivity of 12C6+ in human tumor cells. Cancer Cell Int 2017;17:49.

-32 Ji K, Fang L, Zhao H, Li Q, Shi Y, Xu C, Wang Y, Du L, Wang J, Liu Q: Ginger Oleoresin Alleviated $\gamma$-Ray Irradiation-Induced Reactive Oxygen Species via the Nrf2 Protective Response in Human Mesenchymal Stem Cells. Oxid Med Cell Longev 2017;2017.

-33 Cai J, Fang L, Huang Y, Li R, Yuan J, Yang Y, Zhu X, Chen B, Wu J, Li M: miR-205 targets PTEN and PHLPP2 to augment AKT signaling and drive malignant phenotypes in non-small cell lung cancer. Cancer Res 2013;73:5402-5415. 


\section{Cellular Physiology Cell Physiol Biochem 2018;48:304-316 \begin{tabular}{l|l} 
DOI: 10.1159/000491730 & Ond Biochemistry 2018 The Author(s). Published by S. Karger AG, Basel \\
www.karger.com/cpb
\end{tabular} \\ Ji et al: Sirt1/ NF-kM/Smac Pathway for Radiation Sensitization}

-34 Lee S, Lim MJ, Kim MH, Yu CH, Yun YS, Ahn J, Song JY: An effective strategy for increasing the radiosensitivity of Human lung Cancer cells by blocking Nrf2-dependent antioxidant responses. Free Radic Biol Med 2012;53:807-816.

35 Sun X, Wang Q, Wang Y, Du L, Xu C, Liu Q: Brusatol Enhances the Radiosensitivity of A549 Cells by Promoting ROS Production and Enhancing DNA Damage. Int J Mol Sci 2016;17:

-36 Ko A, Kanehisa A, Martins I, Senovilla L, Chargari C, Dugue D, Marino G, Kepp O, Michaud M, Perfettini JL, Kroemer G, Deutsch E: Autophagy inhibition radiosensitizes in vitro, yet reduces radioresponses in vivo due to deficient immunogenic signalling. Cell Death Differ 2014;21:92-99.

-37 Chang HB, Chen BH: Inhibition of lung cancer cells A549 and H460 by curcuminoid extracts and nanoemulsions prepared from Curcuma longa Linnaeus. Int J Nanomedicine 2015;10:5059-5080.

38 Diehn M, Cho RW, Lobo NA, Kalisky T, Dorie MJ, Kulp AN, Qian D, Lam JS, Ailles LE, Wong M, Joshua B, Kaplan MJ, Wapnir I, Dirbas FM, Somlo G, Garberoglio C, Paz B, Shen J, Lau SK, Quake SR, Brown JM, Weissman IL, Clarke MF: Association of reactive oxygen species levels and radioresistance in cancer stem cells. Nature 2009;458:780-783.

-39 Riley PA: Free Radicals in Biology: Oxidative Stress and the Effects of Ionizing Radiation. Int J of Radiat Biol 2009;65:27-33.

40 Yu H, Liu N, Wang H, Shang Q Jiang P, Zhang Y: Different responses of tumor and normal cells to low-dose radiation. Contemp Oncol (Pozn) 2013;17:356-362.

41 Ciccia A, Elledge SJ: The DNA damage response: making it safe to play with knives. Mol Cell 2010;40:179204.

42 Kuranaga E: Beyond apoptosis: caspase regulatory mechanisms and functions in vivo. Genes Cells 2012;17:83-97.

-43 Chang CJ, Hsu CC, Yung MC, Chen KY, Tzao C, Wu WF, Chou HY, Lee YY, Lu KH, Chiou SH, Ma HI: Enhanced radiosensitivity and radiation-induced apoptosis in glioma CD133-positive cells by knockdown of SirT1 expression. Biochem Biophys Res Commun 2009;380:236-242.

44 Xie Y, Zhang J, Xu Y, Shao C: SirT1 Confers Hypoxia-induced Radioresistance via the Modulation of c-Myc Stabilization on Hepatoma Cells. J Radiat Res 2012;53:44-50.

-45 Hwang S, Guo H-R, Hsieh W, Hwang J-S, Lee S-D, Tang J-L, Chang T-C, Wang J-D, Chang WP: Cancer risk analysis of low-dose radiation exposure. International Congress Series 2007;1299:87-97.

46 Shi YF, Nolan GP: NF- $\kappa B$ to the rescue: RELs, apoptosis and cellular transformation. Trends Genet 1999;15:229-235.

47 Kevin M. Ryan MKE, Nancy R. Rice, Karen H. Vousden: Role of nf-kb in p53-mediated programmed cell death. Nature 2000;404:892-897.

48 Rahman I, Marwick J, Kirkham P: Redox modulation of chromatin remodeling: impact on histone acetylation and deacetylation, NF-kappaB and pro-inflammatory gene expression. Biochem Pharmacol 2004;68:1255-1267.

-49 Wagner L, Marschall V, Karl S, Cristofanon S, Zobel K, Deshayes K, Vucic D, Debatin KM, Fulda S: Smac mimetic sensitizes glioblastoma cells to Temozolomide-induced apoptosis in a RIP1- and NF-kappaBdependent manner. Oncogene 2013;32:988-997.

50 Eckhardt I, Roesler S, Fulda S: Identification of DR5 as a critical, NF-kappaB-regulated mediator of Smacinduced apoptosis. Cell Death Dis 2013;4:e936.

-51 Lin-feng Chen YMaWCG: Acetylation of RelA at discrete sites regulates distinct nuclear functions of NF-kB. EMBO J 2002;21:6539-6548. 